\title{
Crescimento e qualidade de mudas de Inga laurina em função do substrato e adubação suplementar
}

\author{
Murilo Figueredo Campos de Jesus ${ }^{1}$, José Olimpio de Souza Júnior², Gabriel Salles Góes², Ediófila Brito-Rocha², \\ Marcelo Schramm Mielke ${ }^{2 *}$
}

${ }^{1}$ Secretaria de Meio Ambiente do Estado da Bahia, 3 a Avenida Centro Administrativo da Bahia, CEP 41745-005, Salvador, BA, Brasil ${ }^{2}$ Universidade Estadual de Santa Cruz, Rodovia Ilhéus-Itabuna Km 16, CEP 45662-000, Ilhéus, BA, Brasil

\author{
"Autor correspondente: \\ msmielke@uesc.br
}

Termos para indexação: Índice de qualidade de Dickson Taxa de crescimento relativo Restauração florestal

Index terms:

Dickson quality index

Relative growth rate

Forest restoration

Histórico do artigo:

Recebido em 21/05/2015

Aprovado em 09/05/2016

Publicado em 30/06/2016

doi: 10.4336/2016.pfb.36.86.917
Resumo - O objetivo deste estudo foi avaliar o efeito de misturas de um substrato comercial e pó de fibra de coco (PFC) no crescimento e na qualidade de mudas de Inga laurina (Sw.) Willd. (Fabaceae). Tubetes de polietileno foram preenchidos com quatro misturas do substrato comercial HS Florestal@ (HSF) e PFC nas proporções: $\mathrm{T} 1=$ $100 \% \mathrm{HSF} ; \mathrm{T} 2=80 \% \mathrm{HSF}+20 \% \mathrm{PFC} ; \mathrm{T} 3=60 \% \mathrm{HSF}+40 \% \mathrm{PFC}$ e T4 = 40\% HSF $+60 \%$ PFC. Os experimentos foram realizados de forma independente, em presença (experimento I) e ausência (experimento II) de adubação suplementar. Foram realizadas avaliações de crescimento e qualidade das mudas ao final dos experimentos, aos 81 dias (experimento I) e 110 dias (experimento II) após a repicagem. Concluiu-se que o uso da adubação suplementar não apresentou diferenças em crescimento e qualidade das mudas de I. laurina até o percentual de $60 \%$ de PFC misturado ao substrato comercial. Sem a adição de adubação suplementar, a mistura com 80\% HSF + 20\% PFC é indicada para a produção de mudas de I. laurina. Além disso, a ausência de adubação suplementar nitrogenada e a adição de PFC ao substrato estimularam a nodulação.

\section{Growth and quality of Inga laurina seedlings as a function of the substrate and additional fertilization}

\begin{abstract}
The aim of this study was to evaluate the effect of mixtures of a commercial substrate and coconut coir dust (CCD) to grow Inga laurina (Sw.) Willd. (Fabaceae) seedlings. Polyethylene tubes were filled with four mixtures of a commercial substrate HS Florestal ${ }^{\circledR}(\mathrm{HSF})$ and CCD in the following proportions: $\mathrm{T} 1=100 \% \mathrm{HSF}, \mathrm{T} 2=80 \%$ $\mathrm{HSF}+20 \% \mathrm{CCD}, \mathrm{T} 3=60 \% \mathrm{HSF}+40 \% \mathrm{CCD}$ and $\mathrm{T} 4=40 \% \mathrm{HSF}+60 \% \mathrm{CCD}$. The experiments were conducted independently, with supplement fertilization (experiment I) and without (experiment II). Seedlings growth and quality were evaluated at the end of the experiments, 81 days (experiment I) and 110 days (experiment II) after transplanting. It was concluded that supplement fertilization presented no differences in the growth and quality of I. laurina seedlings until up to $60 \%$ of PFC mixed with commercial substrate. Without supplement fertilization the mix of $80 \% \mathrm{HSF}+20 \% \mathrm{PFC}$ is indicate for production of I. laurina seedlings. Moreover, the lack of supplement nitrogen fertilization and addition of PFC to the commercial substrate stimulated nodulation.
\end{abstract}

\section{Introdução}

Devido ao atual estado de degradação das áreas originalmente cobertas por Floresta Atlântica na região Nordeste do Brasil, nos últimos anos tem ocorrido um grande aumento do número de projetos de restauração florestal nesta região (Calmon et al., 2011). Pelo fato de que o plantio de mudas é a técnica mais utilizada em detrimento a outras, como o manejo da chuva de sementes ou a regeneração natural (Rodrigues et al., 2009), a demanda por mudas de espécies florestais nativas tem crescido muito nos últimos anos. Em função da grande durabilidade, possibilidade de reaproveitamento, menor área ocupada no viveiro, além da facilidade de movimentação e de transporte, os tubetes plásticos são as principais embalagens utilizadas em viveiros que produzem mudas florestais em larga escala (Carneiro, 1995). 
O sucesso na produção de mudas de boa qualidade em tubetes depende, entre outros aspectos, da adequada composição dos substratos utilizados, os quais devem ser facilmente disponíveis, padronizados, isentos de patógenos e de sementes de plantas invasoras e, preferencialmente, de baixo custo. Além disso, o substrato deve possibilitar a sustentação das mudas, o bom desenvolvimento das raízes e a fácil retirada da muda do tubete. Também são desejáveis bons atributos químicos, em especial $\mathrm{pH}$ e salinidade (condutividade elétrica) e físicos, como baixa densidade, elevada porosidade e alta capacidade de aeração e de retenção de água (Carrijo et al., 2002; Sodré, 2007; Cavalcante et al., 2008; Dias et al., 2009; Souza Júnior et al., 2011).

Atualmente, existe uma grande diversidade de substratos disponíveis no mercado, os quais diferem em seus atributos físicos, químicos, físico-químicos e biológicos. Até pouco tempo, materiais com altos valores de condutividade elétrica, como a fibra de coco, tiveram os seus usos limitados, devido à elevada salinidade. No entanto, hoje este problema foi contornado com uso de fibras oriundas de regiões não costeiras e/ou com processo de lavagem antes do uso como substrato (Sodré, 2007). A região nordeste é a maior produtora de derivados do coqueiro (Cocos nucifera) do Brasil e a fibra de coco é um subproduto de baixo custo e com grande potencial para uso como condicionante de substratos (Carrijo et al., 2002; Souza Júnior et al., 2011). A fibra de coco como condicionante na formulação de substratos foi testada com sucesso na produção de mudas de várias espécies, entre elas Annona crassiflora (Cavalcante et al., 2008), Hancornia speciosa (Dias et al., 2009), Eucalyptus urophylla (Oliveira Júnior et al., 2011) e Theobroma cacao (Souza Júnior et al., 2011).

A importância de espécies arbóreas da família Fabaceae na recuperação de ambientes degradados é amplamente reconhecida, devido à sua capacidade de nodulação para fixação biológica do nitrogênio (Adams et al., 2010; Chaer et al., 2011). Inga laurina (Sw.) Willd. (Fabaceae) é uma espécie arbórea de médio porte (7 m a $10 \mathrm{~m}$ de altura) e ampla distribuição em toda a América do Sul, sendo comum na Floresta Atlântica da região Nordeste do Brasil; ocorrendo geralmente em solos úmidos, acompanhando os cursos dos rios. Por sua capacidade de nodulação para a fixação biológica do nitrogênio, porte, formato de copa globosa, ocorrência natural e por apresentar frutos comestíveis é útil para sistemas agroflorestais, na arborização urbana e para restauração florestal (Matos \& Queiroz, 2009, Souza, 2013).
Nesse artigo são apresentados os resultados obtidos em dois experimentos independentes, com o objetivo de avaliar o efeito de misturas de um substrato comercial e do pó de fibra de coco no crescimento e na qualidade de mudas de I. laurina em tubetes, com e sem adubação suplementar.

\section{Material e métodos}

Foram realizados dois experimentos, nos quais foram testadas quatro combinações de um substrato comercial e de pó de fibra de coco (PFC) como condicionante, todos com (experimento I) e sem (experimento II) adubação suplementar. Os experimentos foram realizados entre 05/04/2012 e 19/08/2012 no viveiro do Instituto Floresta Viva, situado no distrito de Serra Grande, Município de Uruçuca, BA, coordenadas geográficas -14,463020/ -39,045151, Datum SIRGAS 2000.

A região da área de estudo apresenta clima do tipo Af, típico de florestas tropicais, quente e úmido, sem estação seca definida, com precipitação anual média entre $1.500 \mathrm{~mm}$ a $1.750 \mathrm{~mm}$ e precipitação média mensal de $50 \mathrm{~mm}$ a $100 \mathrm{~mm}$ (podendo chegar a $150 \mathrm{~mm}$ ). O viveiro é dotado de cobertura com tela de sombreamento preta de $50 \%$ de luminosidade, com irrigação automática tipo microaspersão, com bicos com vazão efetiva de $36 \mathrm{~L} \mathrm{~h}^{-1}$, área irrigada de $7 \mathrm{~m}^{2}$ por bico e tempo de irrigação de 5 min, em três horários (07:00, 10:00 e 14:30), à exceção dos dias de chuva.

As sementes foram coletadas de 10 árvores matrizes dos fragmentos florestais localizados à beira da rodovia BA-001, trecho Ilhéus - Serra Grande, em vegetação de restinga, com distâncias superiores a $100 \mathrm{~m}$ entre os indivíduos. Após a coleta e processamento, as sementes foram semeadas em areia lavada. Para o experimento I, foram semeadas 4.400 sementes em 05/04/2012 e a repicagem realizada em 14/04/2012 e para o experimento II, foram semeadas 4.840 sementes em 16/04/2012 e a repicagem realizada em $26 / 04 / 2012$. O experimento I foi finalizado em 04/07/2012 e o experimento II em 14/08/2012.

As mudas foram cultivadas em tubetes de polipropileno com $115 \mathrm{~cm}^{3}$ de capacidade. Foram testadas quatro composições do substrato comercial HS Florestal ${ }^{\circledR}$ (HSF) e o condicionante pó de fibra de coco (PFC) da empresa Amafibra. As análises químicas e físicas dos lotes do substrato comercial e do condicionante são apresentadas nas Tabelas 1 e 2 . Foram testadas quatro misturas de substrato: T1 (100\% HSF), T2 $(80 \% \mathrm{HSF}+20 \%$ $\mathrm{PFC}), \mathrm{T} 3(60 \% \mathrm{HSF}+40 \% \mathrm{PFC}) \mathrm{eT} 4(40 \% \mathrm{HSF}+60 \% \mathrm{PFC})$. 
Tabela 1. Análises químicas* do HS Florestal ${ }^{\circledR}$ (HSF) e do pó de fibra de coco (PFC) utilizados nos experimentos.

\begin{tabular}{|c|c|c|c|c|c|c|c|c|c|c|c|c|c|c|c|}
\hline & $\mathrm{pH}$ & $\mathrm{N}^{-\mathrm{NO}_{3}}{ }_{3}^{-}$ & $\mathrm{N}^{-\mathrm{NH}_{4}}{ }^{+}$ & $\mathrm{P}$ & $\mathrm{S}$ & $\mathrm{K}$ & $\mathrm{Ca}$ & $\mathrm{Mg}$ & $\mathrm{B}$ & $\mathrm{Cl}$ & $\mathrm{Cu}$ & $\mathrm{Fe}$ & $\mathrm{Mn}$ & $\mathrm{Na}$ & $\mathrm{Zn}$ \\
\hline & & & & & & & & $\mathrm{L}^{-1}$ & & & & & & --'- & ---- \\
\hline HSF & 4,3 & 80 & 14 & 4 & 158 & 188 & 149 & 77 & 0,1 & 88 & 0,01 & 0,1 & 3,5 & 11 & 0,20 \\
\hline PFC & 6,3 & 9 & 3 & 11 & 4 & 500 & 2 & 3 & 0,1 & 674 & $<0,01$ & 0,1 & 0,1 & 46 & 0,01 \\
\hline
\end{tabular}

* Método holandês (Sonneveld et al., 1974): extrato de $\mathrm{H}_{2} \mathrm{O}$ 1:1,5 v/v; dosagem: $\mathrm{NO}_{3}^{-}$e $\mathrm{NH}_{4}^{-}$(destilação) e demais elementos (ICP-OES).

Tabela 2. Valores de condutividade elétrica (CE), umidade, densidade úmida, densidade seca e capacidade de retenção de água (CRA) do HS Florestal® (HSF) e pó de fibra de coco (PFC) utilizados nos experimentos.

\begin{tabular}{ccccccc}
\hline & $\mathrm{CE}$ & Umidade a $65^{\circ} \mathrm{C}$ & Densidade úmida & Densidade seca & CRA & CRA \\
\hline & $\mathrm{dS} \mathrm{m} \mathrm{mg} \mathrm{kg}^{-1}$ & $\mathrm{~kg} \mathrm{~kg}^{-1}$ & $\mathrm{~kg} \mathrm{~m}^{-3}$ & $\mathrm{~kg} \mathrm{~m}^{-3}$ & $\mathrm{~m}^{-3} \mathrm{~m}^{-3}$ & $\mathrm{~kg} \mathrm{~kg}^{-1}$ \\
$\mathrm{HSF}$ & 1,1 & 0,382 & 620,3 & 383,3 & 0,432 & 0,118 \\
$\mathrm{PFC}$ & 1,0 & 0,496 & 135,0 & 68,0 & 0,437 & 0,677 \\
\hline
\end{tabular}

Nota: Metodologias descritas nas Instruções Normativas n $n^{\circ}$ 17, de 21/05/2007 e $n^{\circ}$ 31, de 23/10/2008 (BRASIL, 2007, 2008): CE, extração 1:5;

CRA, pelo método da mesa de tensão, a $10 \mathrm{~cm}$ de coluna d'água $(1,0 \mathrm{kPa})$.

Durante todo o período de execução dos experimentos, a radiação fotossinteticamente ativa (RFA) no interior do viveiro e a pleno sol foi monitorada por meio de dois sensores de radiação luminosa S-LIA-M003, acoplados a uma estação meteorológica Hobo Station Data Logger (Onset Computer, Massachusetts, USA), sendo um sensor localizado no interior do viveiro e outro acima da cobertura de tela de sombreamento. A temperatura do ar (Ta) e a umidade relativa do ar (UR) foram monitoradas utilizando-se sensores acoplados à mesma estação meteorológica utilizada para a coleta de dados de RFA. A partir dos valores de Ta e UR foi calculado o déficit de pressão de vapor do ar (DPV). Os valores médios da RFA total diária ao longo dos experimentos, no viveiro e a pleno sol foram, respectivamente, 13,2 \pm 3,4 $\mathrm{mol} \mathrm{m}^{-2} \mathrm{dia}^{-1}$ e 32,8 $\pm 8,2 \mathrm{~mol} \mathrm{~m}^{-2} \mathrm{dia}^{-1}$. Os valores médios de Ta média, Ta máxima, Ta mínima e DPV máximo dentro do viveiro e ao longo do experimento foram, respectivamente, $22,2 \pm 1,0{ }^{\circ} \mathrm{C}, 26,8 \pm 1,3{ }^{\circ} \mathrm{C}$, $18,8 \pm 1,4{ }^{\circ} \mathrm{C}$, e $1,0 \pm 0,3 \mathrm{kPa}$.

No experimento I foi realizada a adubação de base com fosfato monoamônico, logo após o enchimento dos tubetes com os substratos, na dose de $150 \mathrm{mg} \mathrm{dm}^{-3}$ de $\mathrm{P}$, na forma de fosfato monoamônico purificado. A partir de cinco dias após a repicagem, foram realizadas adubações de cobertura, semanalmente, com $30 \mathrm{mg} \mathrm{dm}^{-3}$ de $\mathrm{K}$, na forma de cloreto de potássio, e $60 \mathrm{mg} \mathrm{dm}^{-3} \mathrm{de} \mathrm{N}$, na forma de ureia. Todas as adubações foram feitas com soluções diluídas em água, previamente ajustadas para um litro e utilizando $1 \mathrm{~mL}$ de solução por tubete, com auxílio de uma seringa pistola Walmur $50 \mathrm{~mL}$ modelo 3000. Todas as adubações foram feitas em cobertura após a repicagem. Devido à falta de informações sobre adubação de mudas de espécies arbóreas nativas, propagadas em tubetes e substratos comerciais, as doses de fertilizantes aplicadas no experimento I tiveram como base os trabalhos realizados por Souza Júnior et al. $(2008,2011)$ com mudas de cacaueiro (Theobroma cacao L.) produzidas em tubetes, contendo mistura de Plantmax ${ }^{\circledR}$ e PFC como substrato.

No momento da repicagem, foram coletadas dez mudas e avaliadas a área foliar (AF) e a massa seca de raízes (MSR), caules (MSC) e folhas (MSF). A AF foi avaliada por meio de um medidor de área foliar LI-3100 (Li-Cor, inc. Lincoln, Nebraska, USA). A MSR, a MSC e a MSF foram obtidas após secagem em estufa de circulação forçada de ar a $70{ }^{\circ} \mathrm{C}$ até massa constante. Com base nos valores de MSR, MSC e MSF, foi calculada a massa seca total $(\mathrm{MST}=\mathrm{MSR}$ + MSC + MSF). A colheita das mudas foi realizada, respectivamente, para o experimento I e experimento II, aos 81 dias e 110 dias após a repicagem. Avaliou-se o comprimento do caule (C), o diâmetro do coleto (D), a MSR, a MSC, a MSF e a AF. A partir dos valores de MST no início e no final dos experimentos, foi calculada a taxa de crescimento relativo $\left(\mathrm{TCR}=\operatorname{lnMST}_{2}-\operatorname{lnMST}_{1} /\right.$ $\mathrm{T}_{2}-\mathrm{T}_{1}$ ) e a partir dos valores de MST e AF no início e no final dos experimentos, foi calculada a taxa assimilatória líquida $\left(\mathrm{TAL}=\left(\left(\mathrm{MST}_{2}-\mathrm{MST}_{1}\right) /\left(\mathrm{T}_{2}-\mathrm{T}_{1}\right)\right) \times\left(\left(\ln \mathrm{AF}_{2}-\right.\right.\right.$ $\left.\left.\left.\ln \mathrm{AF}_{1}\right) /\left(\mathrm{AF}_{2}-\mathrm{AF}_{1}\right)\right)\right)$, segundo Hunt (1990).

Também foram calculadas a razão de área foliar $(\mathrm{RAF}=\mathrm{AF} / \mathrm{MST})$, segundo Hunt (1990), e o índice de qualidade de Dickson (IQD = MST/(C/D + MSPA/ MSR)), segundo (Dickson et al., 1960).

No momento da coleta, as mudas do experimento II apresentaram muitos nódulos de bactérias fixadoras de nitrogênio (bactérias diazotróficas) nas raízes. Assim, todos os nódulos de cada tratamento foram 
coletados e colocados em placas de Petri identificadas. Os nódulos foram pesados para a obtenção da massa fresca de nódulos (MFN) e colocados em sacos de papel identificados, e secos em estufa de circulação forçada de ar a $75^{\circ} \mathrm{C}$ até valor constante, para a obtenção da massa seca de nódulos (MSN).

Para os dois experimentos adotou-se o delineamento em blocos ao acaso generalizados (Addelman, 1969), com quatro blocos e três repetições por bloco, sendo a unidade experimental constituída por três mudas. Para a análise da MFN e da MSN, no experimento II, a unidade experimental foi composta por nove mudas. Os resultados obtidos nos dois experimentos foram submetidos à análise da variância, seguida do teste de Tukey a 5\% de probabilidade.

\section{Resultados e discussão}

No experimento I, com adubação suplementar, não foram verificadas diferenças significativas entre tratamentos para nenhuma das variáveis analisadas (Tabela 3). Por outro lado, no experimento II, com ausência de adubação suplementar, o tratamento composto de $80 \% \mathrm{HSF}+20 \%$ PFC (T2) foi significativamente superior aos demais para TCR, TAL e IQD, não diferindo significativamente do tratamento composto de $100 \%$ HSF (T1) para C, D e AF. Assim como ocorreu no experimento I, não foram verificadas diferenças significativas entre tratamentos do experimento II para a RAF.

Diferenças significativas entre tratamentos sem adubação suplementar (experimento II) são indicativos de uma possível deficiência nutricional das mudas à medida que foi adicionado o PFC ao substrato comercial. De fato, de acordo com a análise química realizada antes de serem feitas as misturas e o preenchimento dos tubetes (Tabela 1), o substrato HSF utilizado apresentou em torno de sete vezes mais nitrogênio $(\mathrm{N}), 79$ vezes mais cálcio $(\mathrm{Ca})$ e 23 vezes mais magnésio $(\mathrm{Mg})$ do que o PFC, apesar do PFC apresentar três vezes mais fósforo $(\mathrm{P})$ e três vezes mais potássio $(\mathrm{K})$ do que o HSF. Do ponto de vista do fornecimento de nutrientes essenciais, além de sua menor fertilidade, o PFC é um substrato com baixa densidade de cargas (negativas e positivas), o que propicia elevada lixiviação de nutrientes (Souza Júnior et al., 2008), favorecendo a carência nutricional das mudas, caso não haja a reposição frequente dos nutrientes requeridos em maior quantidade.
Apesar da necessidade de reposição de nutrientes por meio de adubações suplementares, o PFC pode proporcionar inúmeras vantagens para a produção de mudas em viveiros comerciais. Do ponto de vista de seus atributos físicos, a fibra de coco apresenta baixa densidade, elevada porosidade e elevada capacidade de aeração (Abad, 2005), além de não apresentar substâncias fitotóxicas e proporcionar boa aderência das raízes ao substrato, facilitando a retirada da muda do tubete (Noguera, 2000; Carrijo et al., 2002; Correia et al., 2003; Souza Júnior et al., 2011). De fato, para a maioria das variáveis analisadas nos dois experimentos, os melhores resultados não foram obtidos no tratamento com $100 \%$ de HSF (T1), mas sim no tratamento T2, com a adição de $20 \%$ de PFC. Esses resultados indicam que, mesmo sem adubação suplementar, houve melhoria do substrato comercial com a adição de PFC até $20 \%$, possivelmente em atributos físicos no que se refere à densidade e capacidade de retenção de água (Tabela 2).

A TCR e a TAL são variáveis utilizadas em análise do crescimento de plantas, sendo que a TCR relaciona o incremento em biomassa a partir da biomassa préexistente e a TAL relaciona o incremento em biomassa a partir da área foliar (Hunt, 1990). Os valores de TCR obtidos nesse estudo foram relativamente semelhantes entre os dois experimentos e próximos aos valores obtidos por Góes et al. (2015) para mudas de I. laurina, utilizando uma mistura de $90 \%$ HSF e 10\% PFC, com adubação suplementar. Os valores médios da TAL obtidos no experimento I (com adubação suplementar), por outro lado, foram inferiores aos valores obtidos no experimento II (sem adubação suplementar) (Tabela 3 ) e aos valores relatados por Góes et al. (2015). No caso da TAL, as diferenças verificadas entre os experimentos podem estar relacionadas com o aumento da capacidade fotossintética das folhas, em função do tempo de permanência no viveiro, visto que a TAL apresenta relação direta com a taxa fotossintética líquida (Montgomery, 2004; Feijó et al., 2009). Mazzanatti et al. (2015) observaram que a taxa fotossintética líquida aumentou com o tempo de permanência das mudas no viveiro até aproximadamente 100 dias, após o início do experimento para Heliocarpus papayanensis Kunth (Malvaceae), embora não tenham sido encontradas alterações para mudas de Guazuma ulmifolia Lam. (Malvaceae) e Aegiphila integrifolia (Jacq.) Moldenke (Lamiaceae). 
Tabela 3. Comprimento do caule (C), diâmetro do coleto (D), área foliar (AF), razão de área foliar (RAF), taxa de crescimento relativo (TCR), taxa assimilatória líquida (TAL) e índice de qualidade de Dickson (IQD) de mudas de $I$. laurina em função de diferentes proporções de HS Florestal ${ }^{\circledR}$ (HSF) e pó de fibra de coco (PFC), com (experimento I) e sem (experimento II) adubação suplementar.

\begin{tabular}{|c|c|c|c|c|c|}
\hline \multirow[b]{2}{*}{ Variáveis } & \multirow[b]{2}{*}{ Experimento } & \multicolumn{4}{|c|}{ Tratamentos } \\
\hline & & T1 & $\mathbf{T} 2$ & T3 & T4 \\
\hline \multirow{2}{*}{$\mathrm{C}(\mathrm{cm})$} & I & $11,9 \pm 0,44 \mathrm{a}$ & $12,6 \pm 0,81 \mathrm{a}$ & $12,0 \pm 0,68 \mathrm{a}$ & $11,7 \pm 0,87 \mathrm{a}$ \\
\hline & II & $13,8 \pm 0,73 \mathrm{ab}$ & $14,2 \pm 0,85 \mathrm{a}$ & $11,3 \pm 0,73 \mathrm{c}$ & $12,3 \pm 0,43 \mathrm{bc}$ \\
\hline \multirow{2}{*}{$\mathrm{D}(\mathrm{mm})$} & I & $2,5 \pm 0,15 \mathrm{a}$ & $2,8 \pm 0,17 \mathrm{a}$ & $2,5 \pm 0,19 \mathrm{a}$ & $2,5 \pm 0,14 \mathrm{a}$ \\
\hline & II & $2,9 \pm 0,13 \mathrm{a}$ & $2,8 \pm 0,14 \mathrm{ab}$ & $2,6 \pm 0,13 b c$ & $2,4 \pm 0,13 \mathrm{c}$ \\
\hline \multirow{2}{*}{$\mathrm{AF}\left(\mathrm{cm}^{2}\right)$} & I & $56,6 \pm 6,44 \mathrm{a}$ & $56,4 \pm 9,63 \mathrm{a}$ & $47,5 \pm 8,58 \mathrm{a}$ & $46,5 \pm 7,39 \mathrm{a}$ \\
\hline & II & $44,0 \pm 3,63 \mathrm{ab}$ & $48,9 \pm 5,84 \mathrm{a}$ & $35,6 \pm 4,07 \mathrm{~b}$ & $35,7 \pm 4,17 b$ \\
\hline \multirow{2}{*}{$\operatorname{RAF}\left(\mathrm{cm}^{2} \mathrm{~g}^{-1}\right)$} & I & $95,9 \pm 7,66 \mathrm{a}$ & $97,6 \pm 12,71 \mathrm{a}$ & $96,1 \pm 11,74 \mathrm{a}$ & $90,8 \pm 7,47 \mathrm{a}$ \\
\hline & II & $63,7 \pm 4,37 \mathrm{a}$ & $56,8 \pm 3,03 \mathrm{a}$ & $57,9 \pm 9,85 \mathrm{a}$ & $62,6 \pm 3,86 \mathrm{a}$ \\
\hline \multirow{2}{*}{ TCR (mg g $\left.{ }^{-1} \operatorname{dia}^{-1}\right)$} & I & $21,7 \pm 1,43 \mathrm{a}$ & $21,4 \pm 1,39 \mathrm{a}$ & $20,1 \pm 1,76 \mathrm{a}$ & $20,6 \pm 1,36 \mathrm{a}$ \\
\hline & II & $23,5 \pm 0,79 \mathrm{~b}$ & $25,3 \pm 1,04 \mathrm{a}$ & $23,2 \pm 1,12 b c$ & $21,6 \pm 0,98 \mathrm{c}$ \\
\hline \multirow{2}{*}{ TAL $\left(\mathrm{mg} \mathrm{cm}{ }^{-2} \mathrm{dia}^{-1}\right)$} & I & $0,24 \pm 0,03 \mathrm{a}$ & $0,24 \pm 0,03 \mathrm{a}$ & $0,23 \pm 0,03 \mathrm{a}$ & $0,25 \pm 0,03 \mathrm{a}$ \\
\hline & II & $0,34 \pm 0,03 \mathrm{~b}$ & $0,40 \pm 0,03 \mathrm{a}$ & $0,41 \pm 0,07 \mathrm{a}$ & $0,32 \pm 0,02 \mathrm{~b}$ \\
\hline \multirow{2}{*}{ IQD } & I & $0,08 \pm 0,02 \mathrm{a}$ & $0,08 \pm 0,02 \mathrm{a}$ & $0,07 \pm 0,02 \mathrm{a}$ & $0,07 \pm 0,01 \mathrm{a}$ \\
\hline & II & $0,10 \pm 0,01 \mathrm{~b}$ & $0,13 \pm 0,02 \mathrm{a}$ & $0,10 \pm 0,01 \mathrm{bc}$ & $0,08 \pm 0,01 \mathrm{c}$ \\
\hline
\end{tabular}

Tratamentos: T1 (100\% HSF), T2 (80\% HSF + 20\% PFC), T3 (60\% HSF + 40\% PFC) e T4 (40\% HSF + 60\% PFC). Médias \pm erro padrão. Médias seguidas da mesma letra nas linhas não diferem entre si pelo teste de Tukey a $5 \%$ de probabilidade.

Não foram encontrados nódulos nas plantas do experimento I, demonstrando que a adubação inibiu a formação dos mesmos. No experimento II (Figura 1) foi possível observar um aumento significativo na MFN à medida que foi adicionado o PFC ao substrato comercial. Embora não tenham sido verificadas diferenças significativas entre tratamentos para a MSN, observa-se que o valor médio da MSN foi $81 \%$ maior no tratamento $60 \% \mathrm{HSF}+40 \%$ PFC (T3) em comparação ao tratamento com $100 \%$ HSF (T1).

De acordo com Dumroese et al. (2009), plantas não inoculadas e não fertilizadas podem apresentar nódulos. Isso pode ocorrer em ambientes onde as condições de assepsia dos experimentos não são totalmente controladas, como no caso de viveiros florestais, e a contaminação com bactérias nativas pode ocorrer pela deposição de partículas levadas pelo vento, pela ação humana, de outros animais ou por eventos de precipitação pluvial e irrigação (Moreira, 1997; Dumroese et al., 2009). Além disso, é bem conhecido o fato de que a fertilização nitrogenada inibe a formação de nódulos (Patreze \& Cordeiro, 2005). Isso explica o fato de não terem sido observados nódulos no experimento I e o aumento de nódulos à medida que foi adicionado o PFC ao HSF, ou seja, melhoria dos atributos físicos do substrato.

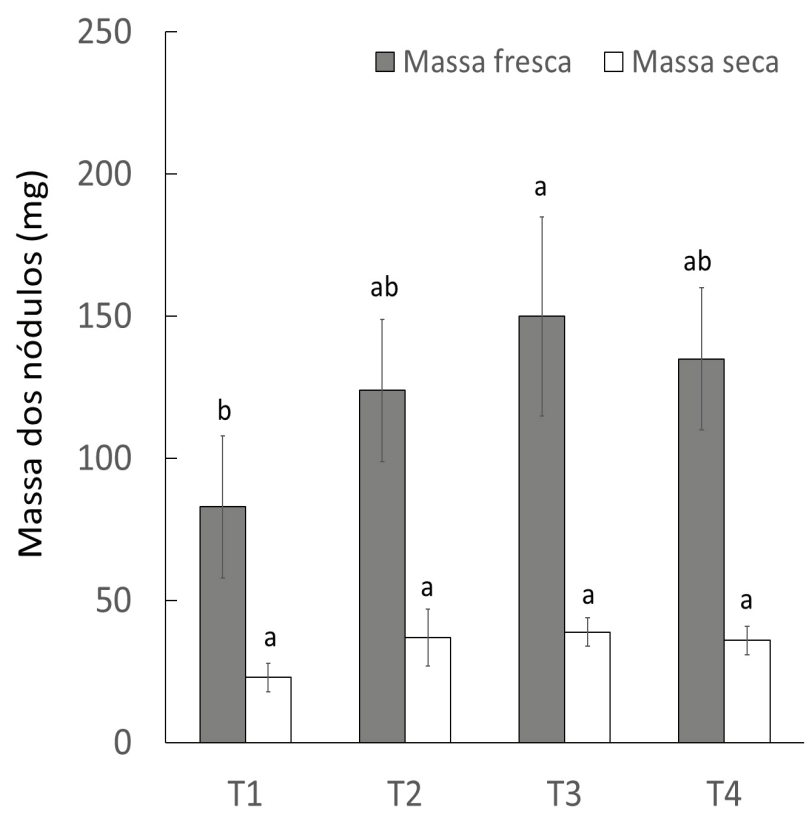

Figura 1. Massa fresca e seca dos nódulos em raízes de mudas de I. laurina em função de diferentes proporções de HS Florestal ${ }^{\circledR}(\mathrm{HSF})$ e pó de fibra de coco (PFC), sem adubação suplementar (Experimento II). Tratamentos: T1 (100\% HSF), T2 (80\% HSF + 20\% PFC), T3 (60\% HSF + 40\% PFC) e T4 (40\% HSF + 60\% PFC). Médias \pm erro padrão. Médias seguidas da mesma letra não diferem entre si pelo teste de Tukey a 5\% de probabilidade. 
A ausência de diferenças significativas entre tratamentos no experimento I, os resultados obtidos no experimento II, além da grande disponibilidade regional de PFC, são indicativos da viabilidade deste condicionante em misturas com substratos comerciais para a produção de mudas florestais, diminuindo assim os custos de produção. Além disso, quando o PFC foi usado em porcentagens mais baixas (20\%) permitiu a formação de nódulos em uma maior qualidade das mudas, sem a necessidade de adubação. Outro aspecto a ser considerado está relacionado com as características físicas do PFC. Quando adicionado aos substratos comerciais, como o HSFlorestal ${ }^{\circledR}$, o PFC pode melhorar as características físicas dos mesmos, como a diminuição da densidade e o aumento da capacidade de retenção de água. Assim, com o objetivo de aperfeiçoar a produção de mudas de espécies arbóreas nativas de boa qualidade, sugere-se a realização de novos estudos sobre o uso do PFC como condicionante de substratos comerciais abrangendo outras espécies florestais nativas, tal qual já foi testado com espécies arbóreas cultivadas (Souza Júnior et al., 2008, 2011).

\section{Conclusões}

Com o uso de adubação suplementar não foram observadas diferenças no crescimento e na qualidade das mudas de Inga laurina até o percentual de $60 \%$ de pó de fibra de coco misturado ao substrato comercial. Sem adubação suplementar, a mistura com $20 \%$ de pó de fibra de coco é indicada para a produção de mudas de $I$. laurina. Além disso, a ausência de adubação suplementar nitrogenada estimulou a nodulação.

\section{Agradecimentos}

Ao CNPq, pelas bolsas de Produtividade em Pesquisa e Desenvolvimento Tecnológico Industrial (DTI-C) a Marcelo S. Mielke e Murilo F. C. de Jesus, respectivametne. Também, aos técnicos do Viveiro Comunitário Floresta Viva Gerson J. Sales Neto, Nilson A. dos Santos e Rones F. Souza pelo auxílio durante a instalação do experimento e a coleta de dados. Essa pesquisa foi financiada pelo $\mathrm{CNPq}$ - Processo 561933/2010-3.

\section{Referências}

Abad, M. et al. Physical properties of various coconut coir dusts compared to peat. Hortscience, v. 40, n. 7, p. 2138-2144, 2005.

Adams, M. A. et al. Woody legumes: a (re)view from the South. Tree Physiology, v. 30, p. 1072-1082, 2010. DOI: 10.1093/treephys/ tpq061.

Addelman, S. The generalized randomized block design. The American Statistician, v. 23, n. 4, p. 35-36, 1969. DOI: 10.1080/00031305.1969.10481868.

Brasil. Instrução Normativa MAPA/SDA n ${ }^{\circ} 17$, de 21 de maio de 2007. Aprova os métodos analíticos oficiais para análise de substratos e condicionadores de solos. Diário Oficial [da] República Federativa do Brasil, Brasília, DF, n. 99, p. 8-9, 24 maio 2007.

Brasil. Instrução Normativa MAPA/SDA n ${ }^{\circ} 31$, de 23 de outubro de 2008. Altera os subitens 3.1.2, 4.1 e 4.1.2 da Instrução Normativa MAPA/SDA n.17 de 21/05/2007. Diário Oficial [da] República Federativa do Brasil, Brasília, DF, n. 207, p. 20, 24 out. 2008.

Calmon, M. et al. Emerging threats and opportunities for large-scale ecological restoration in the Atlantic Forest of Brazil. Restoration Ecology, v. 19, n. 2, p. 154-158, 2011. DOI: 10.1111/j.1526100X.2011.00772.x.

Carneiro, J. G. A. Produção e controle de qualidade de mudas florestais. Curitiba: UFPR/FUPEF, 1995. $451 \mathrm{p}$.

Carrijo, O. A. et al. Fibra da casca do coco verde como substrato agrícola. Horticultura Brasileira, v. 20, p. 533-553, 2002. DOI: 10.1590/S0102-05362002000400003.

Cavalcante, T. R. M. et al. Diferentes ambientes e substratos na formação de mudas de araticum. Revista Brasileira de Fruticultura, v. 30, p. 235-240, 2008. DOI: 10.1590/S0100-29452008000100043.

Chaer, G. M. et al. Nitrogen-fixing legume tree species for the reclamation of severely degraded lands in Brazil. Tree Physiology, v. 31, p. 139-149, 2011. DOI: 10.1093/treephys/tpq116.

Correia, D. et al. Uso de pó de casca de coco na formulação de substratos para formação de mudas enxertadas de cajueiro anão precoce. Revista Brasileira de Fruticultura, v. 25, n. 3, p. 557-558, 2003. DOI: 10.1590/S0100-29452003000300052.

Dias, T. J. et al. Desenvolvimento e qualidade nutricional de mudas de mangabeiras cultivadas em substratos contendo fibra de coco e adubação fosfatada. Revista Brasileira de Fruticultura, v. 31, p. 512-523, 2009

Dickson, A. et al. Quality appraisal of white spruce and white pine seedling stock in nurseries. Forest Chronicle, v. 36, p. 10-13, 1960. DOI: $10.5558 / \mathrm{tfc} 36010-1$.

Dumroese, R. K. et al. Inoculating Acacia koa with Bradyrhizobium and applying fertilizer in the nursery: Effects on nodule formation and seedling growth. HortScience, v. 44, n. 2, p. 443-446, 2009. 
Feijó, N. S. A. et al. Growth and photosynthetic responses of Gallesia integrifolia (Spreng.) Harms and Schinus terebinthifolius Raddi seedlings in dense shade. Agroforestry Systems, v. 77, p. 49-58, 2009. DOI: $10.1007 / \mathrm{s} 10457-008-9190-\mathrm{x}$.

Góes, G. S. et al. Efeitos da inoculação com bactérias diazotróficas e da adubação nitrogenada no crescimento e na qualidade de mudas de Inga laurina (SW.) Willd. (Fabaceae). Revista Árvore, v. 39, n. 6, p. 1031-1038, 2015. DOI: 10.1590/0100-67622015000600005.

Hunt, R. Basic growth analysis. Londres: Unwin Hyman, 1990. $112 \mathrm{p}$.

Matos, E. \& Queiroz, L. P. Árvores para cidades. Salvador: Ministério Público do Estado da Bahia, 2009. 340 p.

Mazzanatti, T. et al. Light acclimation in nursery: morphoanatomy and ecophysiology of seedlings of three light-demanding neotropical tree species. Brazilian Journal of Botany, v. 39, n. 1, p. 19-18, 2015. DOI: $10.1007 / \mathrm{s} 40415-015-0203-5$.

Montgomery, R. Relative importance of photosynthetic physiology and biomass allocation for tree seedling growth across a broad light gradient. Tree Physiology, v. 24, p. 155-167, 2004.

Moreira, F. M. S. Nodulação e crescimento de 49 leguminosas arbóreas nativas da Amazônia em viveiro. Revista Brasileira de Ciência do Solo, v. 21, p. 581-590, 1997. DOI: 10.1590/S010006831997000400008.

Noguera, M. P. Caracterización y evaluación del residuo de fibra de coco: un nuevo material para el cultivo en substrato. 2000. $226 \mathrm{f}$. Thesis (Doctor of Chemistry) - Universidad Politécnica de Valencia, Valencia.

Oliveira Júnior, A. O. et al. Características morfofisiológicas associadas à qualidade de mudas de Eucalyptus urophylla produzidas em diferentes substratos. Revista Árvore, v. 35, n. 6, p. 1173-1180, 2011. DOI: 10.1590/S0100-67622011000700003.
Patreze, C. M. \& Cordeiro, L. Nodulation, arbuscular mycorrhizal colonization and growth of some legumes native from Brazil. Acta Botanica Brasilica, v. 19, n. 3, p. 527-537, 2005. DOI: 10.1590/ S0102-33062005000300014.

Rodrigues, R. R. et al. On the restoration of high diversity forests: 30 years of experiences in the Brazilian Atlantic Forest. Biological Conservation, v. 142 , n. 6 , p. 1242-1251, 2009. DOI: $10.1016 / \mathrm{j}$. biocon.2008.12.008.

Sodré, G. A. Substratos e estaquia na produção de mudas de cacaueiro. 2007. 93 f. Tese (Doutorado em Agronomia) Universidade Estadual Paulista, Jaboticabal.

Sonneveld, C. et al. Analysis of growing media by means of a 1:11/2 volume extract. Communications in Soil Science and Plant Analysis, v. 5, n. 3, p. 183-202, 1974. DOI: 10.1080/00103627409366497.

Souza Júnior, J. O. et al. Características químicas do lixiviado na fase de enraizamento de estacas de cacau em substratos adubados com fósforo. Revista Brasileira de Ciência do Solo, v. 32, n. 4, p. 1573-1581, 2008. DOI: 10.1590/S0100-06832008000400021.

Souza Júnior, J. O. et al. Substrato e adubação fosfatada para a produção de mudas clonais de cacau. Revista Brasileira de Ciência do Solo, v. 35, n. 1, p. 151-159, 2011. DOI: 10.1590/S010006832011000100014.

Souza, R. F. S. Avaliação da viabilidade de espécies arbóreas nativas da Mata Atlântica utilizadas em restauração florestal no sul da Bahia. 2013. 79 f. Dissertação (Mestrado Profissional em Conservação da Biodiversidade e Desenvolvimento Sustentável) - Escola Superior de Conservação Ambiental e Sustentabilidade, Instituto de Pesquisas Ecológicas, Nazaré Paulista. 
